\title{
(2) OPEN ACCESS \\ Virtual reality in specialist palliative care: a feasibility study to enable clinical practice adoption
}

\author{
Amara Callistus Nwosu $\odot 1,{ }^{1,2}$ Mark Mills, ${ }^{2}$ Simon Roughneen, ${ }^{3}$ \\ Sarah Stanley, ${ }^{2}$ Laura Chapman, ${ }^{2}$ Stephen R Mason ${ }^{4}$
}

- Additional material is published online only. To view please visit the journal online (http://dx.doi.org/10.1136/ bmjspcare-2020-002327)

1 International Observatory on End of Life Care, Lancaster University, Faculty of Health and Medicine, Lancaster, UK

${ }^{2}$ Marie Curie Hospice Liverpool, Liverpool, UK

${ }^{3}$ Academic Palliative and End of Life Care Centre, Liverpool University Hospitals NHS Foundation Trust, Liverpool, UK ${ }^{4}$ Palliative Care Unit, University of Liverpool, Liverpool, UK

\section{Correspondence to}

Dr Amara Callistus Nwosu, International Observatory on End of Life Care, Lancaster University Faculty of Health and Medicine, Lancaster LA1 4YG, Lancashire, UK; a.nwosu@lancaster.ac.uk

Received 27 March 2020 Revised 3 February 2021 Accepted 4 February 202

\section{Check for updates}

(c) Author(s) (or their employer(s)) 2021. Re-use permitted under CC BY-NC. No commercial re-use. See rights and permissions. Published by BMJ.

\footnotetext{
To cite: Nwosu AC, Mills M, Roughneen $\mathrm{S}$, et al. BMJ Supportive \& Palliative Care Epub ahead of print: [please include Day Month Year]. doi:10.1136/ bmjspcare-2020-002327
}

\section{ABSTRACT}

Background The use of virtual reality (VR) is increasing in palliative care. However, despite increasing interest in VR, there is little evidence of how this technology can be implemented into practice.

Aims This paper aims to: (1) explore the feasibility of implementing VR therapy, for patients and caregivers, in a hospital specialist inpatient palliative care unit and a hospice, and (2) to identify questions for organisations, to support VR adoption in palliative care.

Methods The Samsung Gear VR system was used in a hospital specialist palliative inpatient unit and a hospice. Patients and caregivers received VR distraction therapy and provided feedback of their experience. Staff completed a feedback questionnaire to explore their opinion of the usefulness of VR in palliative care. A public engagement event was conducted, to identify questions to support implementation of VR in palliative care settings.

Results Fifteen individuals (12 (80\%) patients and 3 (20\%) caregivers) participated. All had a positive experience. No adverse effects were reported. Ten items were identified for organisations to consider ahead of adoption of VR in palliative care. These were questions about: the purpose of VR; intended population; supporting evidence; session duration; equipment choice; infection control issues; content choice; setting of VR; person(s) responsible for delivery and the maintenance plan.

Conclusions It is feasible to use VR therapy in palliative care; however, further evidence about its efficacy and effectiveness is needed. Palliative care practitioners considering VR use should carefully consider several factors, to ensure that this technology can be used safely and effectively in clinical practice.

\section{BACKGROUND}

Virtual reality (VR) is a computerised technology that uses visual graphics, sounds and other sensory input to create

\section{Key messages}

What was already known?

- The use of Virtual Reality (VR) is increasing in palliative care.

- However, despite increasing interest in VR there is little evidence of how this technology can be implemented into practice.

What are the new findings?

- We conducted a quality improvement project in two UK specialist palliative care inpatient units to explore the feasibility of implementing VR therapy, for patients and caregivers.

- Our data suggests that it is feasible to use VR in hospital and hospice settings.

- VR was well received by patients, caregivers and staff. All participants described a positive experience with no major adverse effects.

- We identified questions for organisations to consider, to support VR adoption in palliative care.

What is their significance?

- Our data suggests that it is feasible to use VR in palliative care.

- Practitioners considering using VR should consider a number of factors, concerning the evidence and practical issues, to ensure that this technology can be used safely and effectively in palliative care.

an interactive computer world. ${ }^{1}$ VR is increasingly used in healthcare for symptom management of several conditions. ${ }^{1-5}$ The use of VR in palliative care is growing for variety of purposes, such as education delivery, ${ }^{67}$ and symptom management in hospital ${ }^{8}$ and hospices. ${ }^{9-12}$ Currently, there is little guidance of how VR should be used in clinical care, ${ }^{13}$ and no information about the organisational requirements (eg, internet connectivity) and system processes (eg, infection 
control) necessary to ensure VR can be used safely, effectively and sustainably.

\section{AIM}

This paper aims to: (1) explore the feasibility of implementing VR therapy, for patients and caregivers, in a hospital specialist inpatient palliative care unit and a hospice, and (2) to identify questions for organisations to support VR adoption in palliative care.

\section{METHODS}

This quality improvement project was conducted according to the Plan, Do, Study and Act (PDSA) quality improvement cycle. ${ }^{14}$ The PDSA cycle was chosen as it is an accepted mechanism of implementing change. The project was done through the Liverpool Global Digital Exemplar (GDE) programme. ${ }^{15}$ The GDE programme is a knowledge-sharing platform developed by the English National Health Service, which enables digitally advanced hospitals to innovate and share knowledge globally. ${ }^{16}$

\section{Planning/organisation}

The project was conducted over 3 months (AugustOctober 2018) in two UK specialist palliative care inpatient units. This included a hospital-based 12-bedded (Academic Palliative Care Unit, Liverpool University Hospitals National Health Service Foundation Trust-LUHFT) and a 20-bedded hospice (Marie Curie Hospice Liverpool-MCHL). Both units provide specialist palliative care services (cancer and non-cancer) to a similar geographical population.

\section{Choice of equipment}

The Samsung Gear VR system was chosen due to its portability and ease of use (online supplemental file 1: Virtual reality equipment requirements). This involved a Samsung Galaxy S8 phone positioned in a headmounted display (https://www.samsung.com/global/ galaxy/gear-vr/\#gear-vr). The foam-face cushion was replaced with a polyurethane cushion (Cusfull) to enable decontamination between participants (via $70 \%$ isopropyl alcohol wipe). Bluetooth headphones (Sony WH-CH500) were connected to the phone to provide audio.

\section{Participant evaluation}

Inpatient admissions (both sites), outpatients (hospice only) and caregivers (both sites) were identified by clinical staff (MM-MCHL; SR-LUHFT) and were offered the opportunity to use the VR system. Participants providing written consent were asked to choose one of three VR experiences and complete an evaluation. The VR experiences were downloaded from the Oculus Gear VR store ${ }^{17}$; these included: (1) a 5 -minute-guided relaxation video of a beach (Relax $\left.\mathrm{VR}^{18}\right)$; (2) a 10-minute-guided meditation through a computer-generated forest (Forest of serenity-St
Giles Hospice ${ }^{19}$ ) or (3) a 5-minute-video rollercoaster ride. ${ }^{20} \mathrm{~A}$ modified version of the 'evaluation of VR intervention questionnaire' (online supplemental file 2: Modified virtual reality intervention questionnaire) was used to record feedback. ${ }^{4}$ Participants were verbally asked the following (by MM and SR): What did you think of the VR? What did you like? Was there anything you did not like? Would you want to use this again?

\section{Staff evaluation}

Staff involved in the project were asked to complete an electronic feedback survey (online supplemental file 3: Healthcare professional feedback questionnaire) to gather their feedback on using VR. The survey was a combination of closed and free-text responses. Staff were asked for feedback on the following issues in VR: helpfulness of VR in clinical practice, what went well, problems, barriers and opportunities for future use.

\section{Public evaluation}

We identified public opinion to VR in palliative care by organising a public engagement event, which provided an opportunity for lay representatives to share their views(conducted in MCHL, September 2019). We first presented the project results to the group and then we used a modified world café method ${ }^{21}$ to ask the attendees the following question: 'what questions can organisations use to support VR adoption in palliative care?' A facilitator (ACN) promoted discussion through open questions, and a scribe (SS) collected written feedback.

\section{RESULTS}

Fifteen people participated in the evaluation (table 1). This consisted of $12(80 \%)$ patients and $3(20 \%)$ caregivers. Median age of participants was 63 years $(\mathrm{SD} \pm 16.50)$. The majority were men $(\mathrm{n}=9,60 \%)$. Cancer was the most common diagnosis for patient participants $(n=10 ; 83.3 \%)$. Most people were from the hospice inpatient setting $(\mathrm{n}=7,46.7 \%)$ followed by hospital $(n=6,40 \%)$ and outpatients, respectively $(\mathrm{n}=2,13.3 \%)$.

Relaxation was the most common reason for using VR $(n=11,73.3 \%)$. The beach $(n=7,46.7 \%)$ and forest experiences $(\mathrm{n}=7,46.7 \%)$ were most popular. Most participants had a positive experience of the VR $(\mathrm{n}=14,93.3 \%)$. All participants indicated that they would like to use the VR again. No major complications were noted; although, two participants (13.3\%) reported minor problems (heaviness of the headset, difficulty in adjusting the head straps and problems focusing the image).

Six people (lay representatives) participated in the public engagement event. We identified ten questions to support adoption of VR in palliative care settings, which consisted of the following: the purpose of VR; intended population; supporting evidence; session 


\begin{tabular}{|c|c|c|c|c|c|}
\hline Participant demographic & $N(\%)$ & VR characteristic & $\mathrm{N}(\%)$ & VR characteristic & $\mathrm{N}(\%)$ \\
\hline Median age, years ( \pm SD) & $63.0( \pm 16.50)$ & Setting & & Experience of using VR & \\
\hline Male & $9(60)$ & Hospice inpatient & $7(46.7)$ & Good & $14(93.3)$ \\
\hline \multirow[t]{2}{*}{ Female } & $6(40)$ & Hospice outpatient & $2(13.3)$ & Indifferent & $1(6.7)$ \\
\hline & & Hospital & $6(40)$ & Poor experience & $0(0)$ \\
\hline \multicolumn{6}{|l|}{ Participants } \\
\hline Patients & $12(80)$ & Reason for VR & & Adverse events & \\
\hline \multirow[t]{2}{*}{ Caregivers } & $3(20)$ & Relaxation & $11(73.3)$ & Yes & $0(0)$ \\
\hline & & Pain & $2(13.3)$ & No & $15(100)$ \\
\hline Patient diagnosis, $n=12$ & & Boredom alleviation & $1(6.7)$ & & \\
\hline Cancer & $10(83.3)$ & Anxiety & $1(6.7)$ & Problems with VR use? & \\
\hline Amyloidosis & $1(8.3)$ & & & Yes & $2(13.3)$ \\
\hline \multirow[t]{10}{*}{ Neurological } & $1(8.3)$ & Choice of VR experience & & No & $13(86.7)$ \\
\hline & & Beach & $7(46.7)$ & & \\
\hline & & Forest & $7(46.7)$ & Would they use VR again? & \\
\hline & & Rollercoaster & $1(6.7)$ & Yes & $15(100)$ \\
\hline & & & & No & $0(0)$ \\
\hline & & Time VR used & & & \\
\hline & & $5 \mathrm{~min}$ & $3(20)$ & Was VR requested to be used again? & \\
\hline & & $10 \mathrm{~min}$ & $5(33.3)$ & Yes & $2(13.3)$ \\
\hline & & $15 \min$ & $6(40)$ & No & $13(86.7)$ \\
\hline & & $30 \mathrm{~min}$ & $1(6.7)$ & & \\
\hline
\end{tabular}

duration; equipment choice; infection-control issues; content choice; setting of VR; person(s) responsible for delivery and the maintenance plan (online supplemental file 4: Public engagement event discussionquestions for organisations).

Seven staff members completed the feedback survey (online supplemental file 5: Staff perspectives on virtual reality). Most were based in MCHL $(\mathrm{n}=6,85.7 \%)$ and the majority were doctors $(\mathrm{n}=4,57.1 \%)$. All respondents rated VR as helpful, providing high Likert scores of $4(n=4,57.1 \%)$ and $5(n=3,42.9 \%)$. Following the end of the project, further VR use was recommended by five (71.4\%) respondents. All staff stated their willingness to use VR in the future. Free-text responses provided further feedback (online supplemental file 6: Free-text questionnaire responses from health professionals detailing their views about the use of virtual reality in palliative care); in summary, the reported benefits of VR were its ease of use, the improvements in psychological well-being and the observed positive short-term effects in participants. Problems with the VR included the discomfort of the headset, disorientation noted by some participants, and technical issues relating to setting up and charging the device. Barriers to VR use were identified as infection-control issues, issues with staff unfamiliar of how to use the equipment, and technical issues of ensuring the equipment was updated, charged and ready for use. Future possible opportunities to use VR in palliative care were identified, and these included 'distraction therapy' for patients undergoing clinical procedures (eg, ascitic drain insertion), virtual hospice visits, family meetings and therapy sessions.

\section{DISCUSSION}

Our data suggest that it is feasible to use VR in hospital and hospice settings. VR was well received by patients, caregivers and staff. All participants described a positive experience with no major adverse effects. Ten questions were identified for organisations to consider, to support VR adoption in palliative care.

\section{Contribution and strengths of this paper}

This is the first paper in the literature to begin to develop a framework to consider how VR can be implemented in palliative care. This paper is consistent with previous studies which demonstrate the feasibility of using VR in palliative care settings.

\section{Relation to previous work}

The findings of this paper suggest feasibility of VR in hospital palliative care settings. This is consistent with previous work by Niki et $a l,{ }^{8}$ who identified symptomatic improvement for 20 hospital inpatients with advanced cancer. Similarly, our work suggests feasibility of VR in hospice settings, which supports the outcomes of previous studies that demonstrate positive outcomes of VR in hospice populations. ${ }^{9-12}$

For palliative care VR, it is important to consider the purpose of the activity, to identify how content is developed, and to define how (and by whom) it is delivered. Our study used software developed specifically for palliative care ${ }^{19}$ and generic resources. ${ }^{1820}$ To date, no VR resources have been validated for the specific purpose of providing symptom relief in palliative care. Consistent with previous work, our findings report that palliative 
care VR should be evidence based. ${ }^{13}$ Knowledgetransfer considerations to support implementation of VR in palliative care have not been previously reported in the literature. Our study reports on important practical issues, such as choice of VR system, infection-control issues and technical device issues such as storage, charging and maintenance. ${ }^{22}$

\section{Limitations}

Limitations to this project are its small scope and feasibility focus, meaning that no conclusions about the effectiveness and efficacy of VR can be made. A completely immersive experience was not possible from the device; meaning that participants may have a better experience with other systems. ${ }^{10}$ Some participants struggled to independently operate aspects of the VR device and required assistance, demonstrating that technology should be optimised for user requirements. Remote operation and second screen viewing were not possible from this VR device, which meant that the operator needed to stay with the participant for the entirety of the session.

Technical challenges were observed. First, software updates were frequently required, which necessitated planning to ensure the device was updated prior to use. Second, it was necessary to charge the phone and headphones separately, which was occasionally impractical. Finally, internet connectivity problems were encountered which prevented VR use; cellular mobile internet was used in these occasions, which reduced the video quality.

\section{Implications for policy and practice and research}

Our paper highlights a number of practical questions to support organisations considering use of VR in palliative care. Although the clinical use of VR in palliative care appears feasible and safe, further evidence of its benefit, effectiveness and practicality are required before recommendations can be made about its usefulness. Further research is needed to examine whether VR can effectively improve symptom management in palliative care and to ensure its use is practical, meaningful and evidence based.

\section{CONCLUSION}

Our data suggest that it is feasible to use VR in palliative care. Practitioners considering using VR should consider a number of factors, concerning the evidence and practical issues, to ensure that this technology can be used safely and effectively in palliative care.

Twitter Amara Callistus Nwosu@amaranwosu

Acknowledgements ACN's, MM's, LC's and SS's hospice posts are supported by Marie Curie Hospice Liverpool. SS's academic post is supported by funding from the National Institute for Health Research (NIHR) North West CoastClinical Research Network (CRN).

Contributors Study design-ACN, SR and MM. Data collection-MM and SR. Paper writing-ACN. Critique and review of the final manuscript-ACN, MM, SR, SS, LC and SRM.

Funding Liverpool University Hospitals NHS Foundation Trust, Global Digital Exemplar Programme, used to purchase equipment, $£ 2968.6$ (grant number-not applicable). Health and Life Sciences, Faculty Public Engagement Grants Scheme, Wellcome Trust, University of Liverpool, used to fund the patient engagement meeting, $£ 360$ (grant number-not applicable).

Competing interests None declared.

Patient consent for publication Not required.

Provenance and peer review Not commissioned; externally peer reviewed.

Open access This is an open access article distributed in accordance with the Creative Commons Attribution Non Commercial (CC BY-NC 4.0) license, which permits others to distribute, remix, adapt, build upon this work noncommercially, and license their derivative works on different terms, provided the original work is properly cited, appropriate credit is given, any changes made indicated, and the use is noncommercial. See: http://creativecommons.org/licenses/by-nc/4. $0 /$.

\section{ORCID iD}

Amara Callistus Nwosu http://orcid.org/0000-0003-0014-3741

\section{REFERENCES}

1 Chirico A, Lucidi F, De Laurentiis M, et al. Virtual reality in health system: beyond entertainment. A mini-review on the efficacy of VR during cancer treatment. J Cell Physiol 2016;231:275-87.

2 Hajesmaeel Gohari S, Gozali E, Niakan Kalhori SR. Virtual reality applications for chronic conditions management: a review. Med I Islam Repub Iran 2019;33:67.

3 Oyama H. Virtual reality for the palliative care of cancer. Stud Health Technol Inform 1997;44:87-94.

4 Schneider SM, Hood LE. Virtual reality: a distraction intervention for chemotherapy. Oncol Nurs Forum 2007;34:39-46.

5 Oyama H, Kaneda M, Katsumata N, et al. Using the bedside wellness system during chemotherapy decreases fatigue and emesis in cancer patients. J Med Syst 2000;24:173-82.

6 Evans L, Taubert M. State of the science: the doll is dead: simulation in palliative care education. BMJ Support Palliat Care 2019;9:117-9.

7 Lee AL, DeBest M, Koeniger-Donohue R, et al. The feasibility and acceptability of using virtual world technology for interprofessional education in palliative care: a mixed methods study. J Interprof Care 2020;34:461-71.

8 Niki K, Okamoto Y, Maeda I, et al. A novel palliative care approach using virtual reality for improving various symptoms of terminal cancer patients: a preliminary prospective, multicenter study. J Palliat Med 2019;22:702-7.

9 Johnson T, Bauler L, Vos D, et al. Virtual reality use for symptom management in palliative care: a pilot study to assess user perceptions. J Palliat Med 2020;23:1233-8.

10 Popert S, Riat H. P-35 can virtual reality (VR) guided meditation reduce pain? A feasibility and acceptability study. BMJ Support Palliat Care 2017;7:A22.

11 Perna-Forrest L. P-34 unlocking the potential of virtual reality in palliative care. BMJ Support Palliat Care 2017;7:A22.

12 Perna-Forrest L, Minton O. 149 the potential for virtual reality therapy in palliative care-preliminary findings. BMJ Support Palliat Care 2019.

13 Austin P, Lovell M, Siddall P. The efficacy of virtual reality for persistent cancer pain: a call for research. J Pain Symptom Manage 2019;58:e11-14. 
14 ACT Academy - NHS Improvement. Plan, do, study, act (PDSA) cycles and the model for improvement, 2017. Available: https://improvement.nhs.uk/resources/pdsa-cycles/: NHS Improvement

15 Royal Liverpool Global Digital Exemplar. Digital Liverpool, our digital future, 2017. Available: https:// www.rlbuht.nhs.uk/media/5572/digital_liverpool_april 2017.pdf: Liverpool Univerity Hospitals NHS Foundation Trust

16 NHS England. Global digital exemplars, 2018. Available: https://www.england.nhs.uk/digitaltechnology/connecteddigital systems/exemplars/

17 Facebook Technologies. Oculus gear VR store. Available: https://www.oculus.com/experiences/gear-vr/2019 [Available from: https://www.oculus.com/experiences/gear-vr/
18 Relax VR. Relax VR, 2019. Available: https://www.relaxvr.co/ virtualrealityspas

19 St. Giles Hospice. Forest of serenity, 2017. Available: https:// www.stgileshospice.com/forest-of-serenity/

20 Youtube. 3D VR 360 VIDEOS. 360 video VR roller coaster ride 4K, 2018. Available: https://www.youtube.com/watch?v= kxPZfwAM2bE\&feature $=$ youtu.be

21 The World Cafe. World CAFE method website of the world CAFE, 2018. Available: http://www.theworldcafe.com/keyconcepts-resources/world-cafe-method/\#

22 Glegg SMN, Levac DE, Barriers LDE. Barriers, facilitators and interventions to support virtual reality implementation in rehabilitation: a scoping review. $P m$ R 2018;10:1237-51. 